Historic, Archive Document

Do not assume content reflects current scientific knowledge, policies, or practices. 



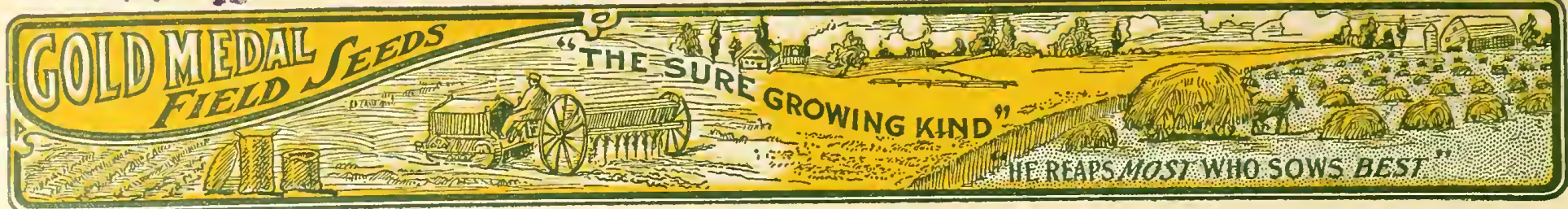

Subject to immediate acceptance and market changes we quote fob Louisville

SEPTEMBER 10, 1929

Rosen

NEW CROP RYE

Northern White...

$\$ 1.40$ per bu.

1.35 per bu.

10 Sax Lots or More, 5c Bushel Less

\section{RED CLOVER}

Gold Medal Domestic . . . . \$ \$24.00

Choice Domestic ... . . . 22.00

Royal Domestic ...... 20.00

Special Small Grain . . . . . 17.50

Best French . . . 20.00

Choice French . . . 17.50

Mammoth or Saplin Clover

Gold Medal . . . . . . \$25.00

Choice .......... 23.00

Royal

22.00

\section{ALSIKE}

Gold Medal . . . . . $\$ 22.50$

Choice ........ 21.00

Specisl, contains White Clover . . . . 20.00

Special, contains Red Clover . . . . . 15.00

\section{ALFALFA}

Gold Medal . . . \$23.00

Choice.

21.00

\section{GRIMMS ALFALFA}

Utah Grown

$\$ 37.50$

Sealed, verified and packed in 30 and $60 \mathrm{lb}$. bags.

\section{SWEET CLOVER}

Gold Medal, W. B. Biennial $\$ 8.00$

Choice. “" " 7.50

Gold Medal, Yellow Biennial 8.50

Choice

8.50
7.50

CRIMSON CLOVER

Domestic Grown . . . . $\$ 15.00$

JAPAN CLOVER

Choice (Recleaned) . . . . \$18.00

\section{WHITE CLOVER}

Gold Medal . . . . . . $\$ 30.00$

Choice . . . 20.00

Special . . . . . 17.50

\section{TIMOTHY}

Gold Medal ... . . . . . \$6.50

Abe (99\% Pure) . . . . 6.25

Choice .......... 5.75

Royal ............. . 5.00

\section{ALSIKE and TIMOTHY}

Choice Quality....... \$7.50

\section{PASTURE MIXTURE}

Contains Timothy, Alfalfa, Alsike,

Redtop, etc. ....\$5.00

With Orchard Grass . . . . 6.50

\section{RED TOP}

Gold Medal . . . . . . \$16.50

Abe $(90 \%$ Pure) $\ldots 16.00$

Choice . . . . . . 15.50

Royal ..... 14.00

Unhulled . ...... 8.50

\section{ORCHARD GRASS}

Gold Medal . . . . . . \$16.00

Choice .... 15.50

Royal . . . . . 14.50

\section{KY. BLUE GRASS}

Gold Medal . . . . . . $\$ 21.00$

Choice . . . 20.00

Light Weight $\ldots \ldots \ldots 12.50$

RYE GRASS

Domestic Recleaned .... . \$8.00
WINTER BARLEY

Bearded .......\$ 1.40

\section{SEED WHEAT}

Blue Stem ........ $\$ 2.00$

\section{LAWN GRASS}

Gold Medal . . . . . . \$28.CO

Cherokee..... 25.00

Shady Place . . . 33.00

Put up in muslin bags printed in two colors, $\mathbf{I} \mathrm{lb}$.,

$3 \mathrm{lb}, 5 \mathrm{lb}$. net. Above grades in bulk $\$ 3.00$ per

cwt. less. Also in I lb. cartons; same price as I lb. bags.

\section{MISCELLANEOUS}

Sudan Grass . . . . . $\$ 5.00$

Buckwheat . . . . . . 4.00

Winter or Hairy Vetch .... 9.50

Dwarf Essex Rap? .... 7.50

Tall Meadow Oat Grass .. 17.00

\section{SEED GRAIN}

Winter Turf Oats . ...\$1.25

Burt Oats ..... $\quad .85$

Feed Oats ........ $\quad .65$

\section{SEED SOWERS}

Cyclone each . . \$1.75 Doz. \$18.00 Horn ". ... .75 Doz. 7.50

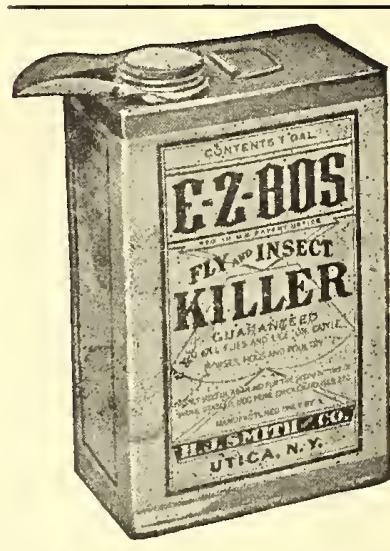

\section{E-Z-BOS Fly and} Insect Killer

Sells the Entire Year KILLS ALL. INSECTS, INCLUDING Lice, Mites, Horse Flies, etc. Well Known Easy Seller Proftable Side Line for any Business

Gallon Cans, per doz. $\$ 12.00$ Retails $\$ 1.50$ each

Half gallon cans, per doz. 7.20 Retails $\$ 1.00$ each

Quart Cans, per doz. . 4.80 Retails $65 \mathrm{c}$ each

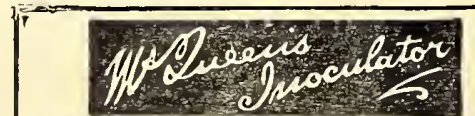

NEW REDUCED PRICES For CLOVERS or ALFALFA Price $_{\substack{\text { Pase } \\ \text { Per can }}}$ (Small Seeds) Per case Per can

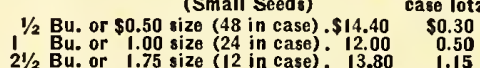
For Soy Beans, Vetch, Peas, Beans, Cow Peas (All Large Seeds) $\begin{array}{lllr}1 & \text { Bu. or } \$ 0.50 \text { size (48 in case) } \ldots . \$ 14.40 & \$ 0.30 \\ 2 \text { Bu. or } 1.00 \text { size (24 in case) } \ldots .12 .00 & 0.50 \\ 5 \text { Bu. or } 1.75 \text { size (12 in case) } \ldots . .13 .80 & 1.15\end{array}$ Less than case lots, add ten cents per can.
Cases May be Assorted. State Seed to be Treated.

\section{.}


47. Barley

... . . 75-100

60 . Beans, Soy, drills ......... . 30- 40

60 . Beans, Soy, broadcast . . . . . . . . . $60-80$

14. Bluegrass, Kentucky . . . . . . . . . 21-28

48. Buckwheat . . . . . . . . . . . . 50- 75

50 . Cane, Drill .. . . . . . . . . . . 10- 15

50 . Cane, broadcast . . . . . . . . . . 50-75

6i). Clover, Alfalfa, drills ........ . . 15- 20

60 . Clover, Alfalfa, broadcast ... . . . . . 18- 22

60 . Clover, Alsike (alone for forage). . . . . . 8- 15

60 . Clover, Alsike (on wheat or rye in spring ). . 4-6

60.. Clover, Crimson ........... 12- 15

25 . Clover, Japan (Lespedeza) . . . . . . . 15- 25

60 . Clover, Sapling (Mammoth) . . . . . 10- 15

60 . Clover, Red (alone for forage) . . . . . . . 10- 15

60 . Clover, Red (on small grain in spring) . . . 7- 12

60. Clover, Sweet (hulled) . . . . . . . . 10- 15

33 .. Clover, Sweet (unhulled) . . . . . . . 20-30

60 . Clover, White ........... . . 10- 20

56 . Corn . . . . . . . . . . . . . 6- 12

56 . . Corn (for Silage) . . . . . . . . . 40-56

50 . Millet, All Varieties (for seed) . . . . . . 25- 40

50 . Millet, All Varieties : for hay! . . . . . . . 40-50

32. Oats.............. . . . 50- 65

14. Orchard Grass . . . . . . . . . . . 20-30

60 . Peas, Cowpeas (drills) ......... . . 45-60

ค. . Peas, Cowpeas (broadcast) . . . . . . . 75- 90

51. . Rape (drill) . . . . . . . . . . . . . 4-8

50. . Rape (broadcast) . . . . . . . . . $8-10$

14. . Red Top Fancy . . . . . . . . . . . 7- 12

14. . Red Top (unhulled) . . . . . . . . 10- 15

56. . Rye . . . . . . . . . . . . . 56-112

25. . Sudan Grass . . . . . . . . . . . . $15-25$

24. Sunflower ............... . $8-10$

45. Timothy .............. . . 10- 15

45 . Timothy and Alsike .......... . . 8- 10

60 . Vetch, Hairy (drills) . . . . . . . . 35- 50

60 . Vetch, Hairy (broajcast) . . . . . . . 50- 75

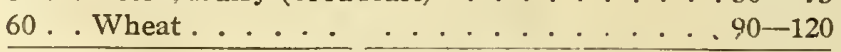

\section{Louisville Seed Company}

226 N. Fifteenth St.

LOUISVILLE, KENTUCKY 